\title{
Fertility-sparing treatment using medroxyprogesterone acetate for endometrial carcinoma
}

\author{
HIROYUKI FUJIWARA ${ }^{1}$, TOSHIKO JOBO ${ }^{2,3}$, YUJI TAKEI ${ }^{1}$, YASUSHI SAGA ${ }^{1}$, MANAMI IMAI ${ }^{2}$, \\ TSUTOMU ARAI $^{2}$, AKIYO TANEICHI ${ }^{1}$, SHIZUO MACHIDA ${ }^{1}$, YOSHIFUMI TAKAHASHI ${ }^{1}$ and MITSUAKI SUZUKI ${ }^{1}$ \\ ${ }^{1}$ Department of Obstetrics and Gynecology, Jichi Medical University, Shimotsuke, Tochigi 329-0498; \\ ${ }^{2}$ Department of Obstetrics and Gynecology, Kitasato University School of Medicine, Sagamihara, Kanagawa 252-0374; \\ ${ }^{3}$ Department of Gynecology, Social Insurance Sagamino Hospital, Sagamihara, Kanagawa 252-0206, Japan
}

Received December 13, 2011; Accepted February 1, 2012

DOI: $10.3892 / \mathrm{ol} .2012 .602$

\begin{abstract}
The purpose of this study was to present the results of fertility-sparing treatment using medroxyprogesterone acetate (MPA) for endometrial carcinoma (EC), and to clarify patient characteristics by investigating patient background factors. A total of 59 patients with EC, who received MPA as fertility-sparing therapy at two institutions over a 21-year period between 1987 and 2008, were studied retrospectively. Patients were administered oral MPA at 400-600 mg/day for 16-24 weeks as long as they responded. Endometrial tissue was assessed twice, at 8-12 weeks (during treatment) and shortly after treatment. The overall complete response (CR) rate was $71 \%$. A total of $22(52 \%)$ of 42 responders later developed relapse. A total of 19 cases became pregnant, and 25 infants were born. Eighty percent of recurrences occurred within 2 years. For stages Ia and Ib-IIa (FIGO, 1988), initial CR rates were 80.0 and $42.9 \%$, respectively $(\mathrm{p}<0.01)$, demonstrating a significant difference. Total hysterectomy was performed for 26 patients (44\%) due to recurrence or failure to respond to the initial treatment. Among these 26 patients, postoperative stages were more advanced in 10 patients (38\%). The grade advanced (became more poorly differentiated) postoperatively in 2 patients (8\%). Premenopausal females with EC can be treated successfully with MPA, however patients should be informed of the risks and limitations of this conservative treatment.
\end{abstract}

\section{Introduction}

In Western countries, endometrial carcinoma (EC) is the most common type of malignant tumor in the field of gynecology $(1,2)$. A state of persistent high estrogen, as observed

Correspondence to: Dr Hiroyuki Fujiwara, Department of Obstetrics and Gynecology, Jichi Medical University, 3311-1 Yakushiji, Shimotsuke, Tochigi 329-0498, Japan

E-mail: fujiwara@jichi.ac.jp

Key words: endometrial carcinoma, fertility-sparing treatment, medroxyprogesterone acetate with ovulation disorders and obesity, is considered a risk factor (3-5). The standard treatments for EC are total hysterectomy and bilateral adnexectomy.

$\mathrm{EC}$ is a disease that frequently affects perimenopausal females, however approximately $10 \%$ of affected females are $\leq 40$ years old, and the incidence in young females has recently been on the increase (6). As in Western countries, the number of $\leq 40$-year-old females with EC in Japan is on the rise (7). Thus, the number of patients who select to undergo fertilitysparing treatment is growing.

Studies at various institutions have reported the results of fertility-sparing treatment for EC using progesterone preparations (8-17). However, as the number of cases have been low, the efficacy of such treatment has yet to be clarified. Furthermore, few studies have closely described the clinical background of young females with early-stage EC wishing to undergo fertility-sparing treatment.

The present study investigated the effects of treatment, prognosis, pregnancy status and other factors, in order to demonstrate the therapeutic outcomes of current fertility-sparing treatment. Furthermore, patient background factors were investigated to elucidate the characteristics of the patient group.

\section{Patients and methods}

Patients. A total of 59 patients with EC who underwent fertilitysparing treatment, at either the Jichi Medical University Hospital or the Kitasato University Hospital over the 21-year period from 1987 to 2007, were retrospectively investigated. Atypical endometrial hyperplasia was excluded in this study. Selection criteria of the treatment were: i) highly differentiated (G1) endometrioid adenocarcinoma; ii) no appearance of myometrial invasion (stage Ia: FIGO, 1988) on magnetic resonance imaging; iii) unmarried or strong desire to have a child; iv) no current or past history of thrombotic disease. As a general rule, blood clotting and fibrinolysis were normal. However, when patients strongly desired, fertility-sparing treatment was performed on patients with stage Ib (in which myometrial invasion may be suspected) or IIa (in which cervical mucosa invasion may be suspected). In all patients, treatment was administered only after written informed 
Table I. Patient characteristics.

Patient characteristics, $n=59$

Median age, years (range)

$31(21-42)$

Mean BMI, $\mathrm{kg} / \mathrm{m}^{2}$ (range)

$23.3(15-38)$

Clinical stage (cases)

Ia

$44(75 \%)$

Ib-IIa

Risk factor (cases)

Irregular periods

$37(63 \%)$

Nulligravida

PCO syndrome

$4(7 \%)$

Detectability (cases)

Metrorrhagia

$35(59 \%)$

Infertility examination

$16(27 \%)$

Abnormal menses ${ }^{\mathrm{a}}$

$6(10 \%)$

${ }^{a}$ Hypermenorrhea/dysmenorrhea/amenorrhea. PCO, polycystic ovary syndrome.

consent was obtained. Pathological specimens were examined by experienced pathologists in each institute.

Treatments. In the fertility-sparing treatment, a dose of 400-600 mg/day of medroxyprogesterone acetate (MPA) was administered orally, and the entire surface was curetted between 8 and 12 weeks to confirm the therapeutic effects and absence of disease progression. Following administration of the drug for 16-24 weeks, the endometrium was again curetted, and therapeutic effects were assessed. At this stage, if the lesion persisted, total hysterectomy was selected as a general rule; if the lesion had disappeared, the patient was monitored and allowed to become pregnant. Even when the lesion persisted, MPA was continued if the patient strongly preferred to preserve fertility. In such patients, pathological tests were conducted every 4-8 weeks, and the duration of therapy was determined individually.

\section{Results}

Patient characteristics. A total of 59 patients, including 44 patients with stage Ia (75\%) and 15 with stage Ib-IIa (25\%) were included in the present study (Table I). The median age was 31 years, and the mean body mass index was $23.3 \mathrm{~kg} / \mathrm{m}^{2}$. The proportions of females with menstrual irregularity, nulliparity and polycystic ovary syndrome (PCO), which are considered risk factors for EC, were 63, 98 and 7\%, respectively. The cause of detection was metrorrhagia in 35 patients (59\%), and incidental discovery of the lesion during screening or treatment for infertility in 16 patients (27\%).

MPA administration positively affects EC patients despite recurrence. In the present study, the mean duration of MPA administration until complete response (CR) was 24.9 weeks (range, 13-70). Fig. 1 shows the therapeutic results. The median duration of follow-up was 66 months (range, 11-251). The response to the initial treatment was $\mathrm{CR}$ in 42 patients $(71 \%)$, and either partial response (PR) or no change (NC) in 17 patients (29\%). In 16 of the 17 patients without CR, total hysterectomy was performed and fertility could not be preserved. One patient

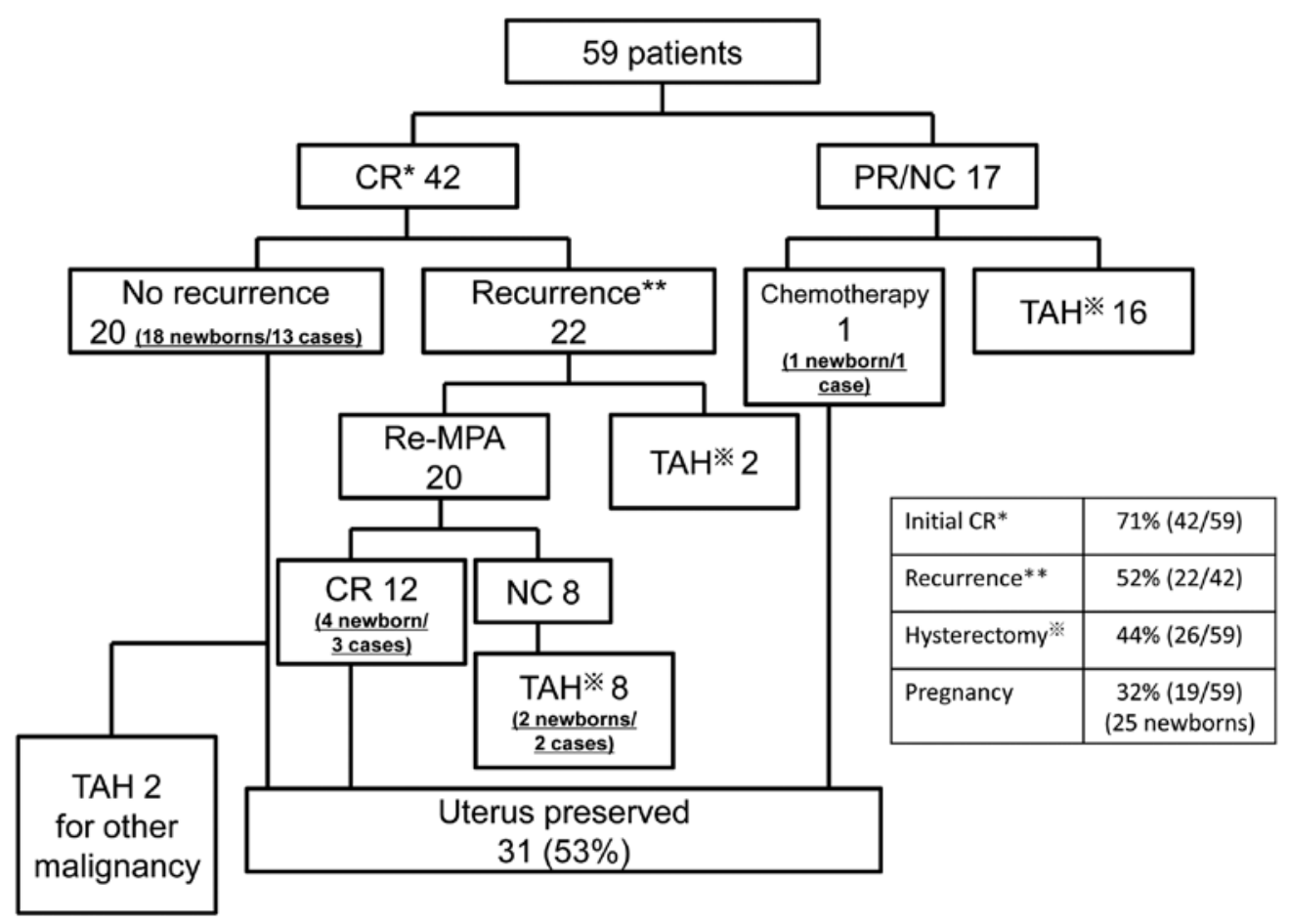

Figure 1. Therapeutic results of fertility-sparing treatment using MPA. MPA, medroxyprogesterone acetate; CR, complete response; PR, partial response; $\mathrm{NC}$, no change; TAH, total abdominal hysterectomy. 
(\%)

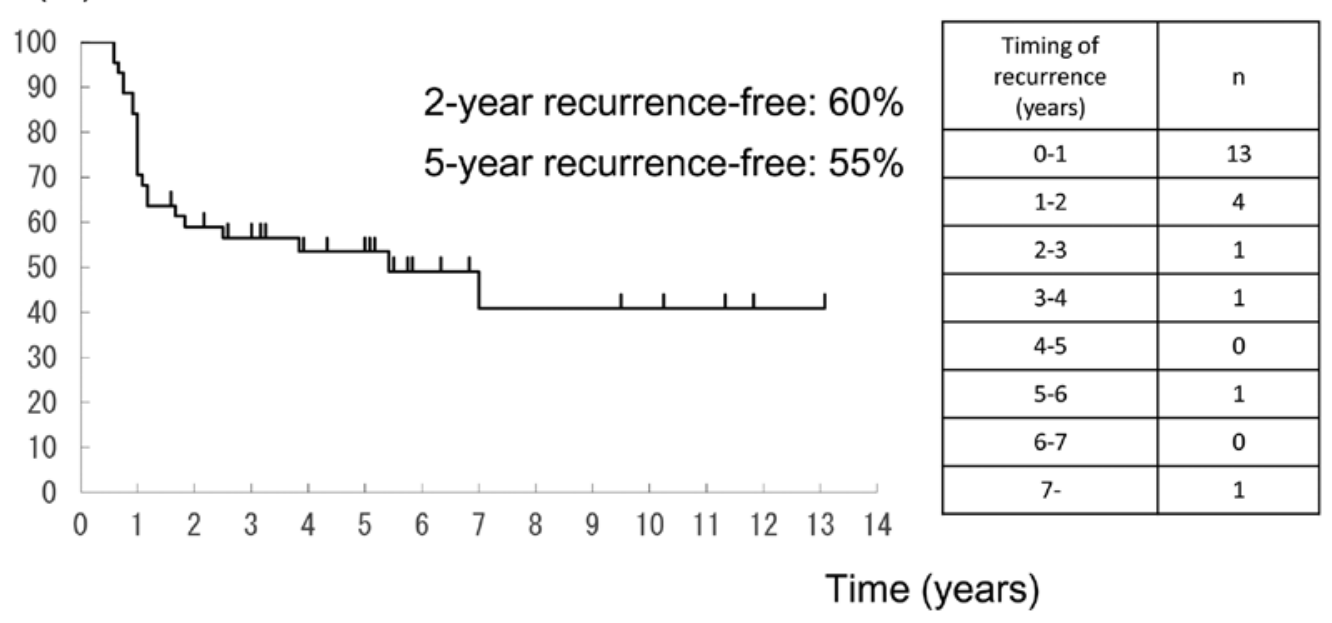

Figure 2. Recurrence-free survival curve and timing of recurrence (Kaplan-Meier analysis).

refused surgery and underwent cytotoxic chemotherapy; subsequently, the cancer went into remission. This patient gave birth to a child and is currently alive and disease-free. Of the 16 patients who underwent surgery, 15 are currently alive and disease-free, but cancer recurred in one patient, who succumbed to recurrence 126 months after initial treatment.

A survey of the 42 initial CR patients revealed that 20 patients $(48 \%)$ remain alive and are disease-free, with a median recurrence-free survival of 62 months (range, 19-157). Of these 20 patients, 13 patients have given birth to a total of 18 children. However, 2 patients subsequently underwent total hysterectomy; one for new ovarian cancer and the other for new tubal cancer.

Recurrence was observed in 22 patients (52\%), with a median onset of recurrence of 12 months (range, 7-84). In 20 of these 22 patients, MPA was again administered, and remission was achieved in 12 patients without recurrence. A total of 3 of these patients have given birth to a total of 4 children. In 8 patients, CR could not be achieved despite additional MPA administration, and total hysterectomy was performed. However, 2 of these 8 patients had each given birth to a child prior to recurrence. Of the 22 patients with recurrence, 2 patients stopped visiting the hospital and have not been followed up.

Fig. 2 shows the timing of recurrence following remission and the recurrence-free survival curve. In 18 (82\%) of the 22 patients who had recurrence, cancer recurred within 2 years, and the longest time to recurrence was 7 years and 1 month.

The initial $C R$ rate and the rate of recurrence following $C R$ in relation to the clinical stage. For patients with stage Ia, the CR rate was significantly higher than that for the other stages ( 80.0 vs. $42.9 \%$, respectively; $\mathrm{p}<0.01$ ). The rate of recurrence was lower for stage Ia patients than recurrence for other stages (47.2 vs. $83.3 \%$, respectively; $\mathrm{p}=0.11$ ), however there was no statistically significant difference (Fig. 3).

Following exclusion of the 2 patients who underwent total hysterectomy for other types of cancer, the 26 patients who underwent total hysterectomy for the primary disease were examined. Clinical and pathological stages matched in

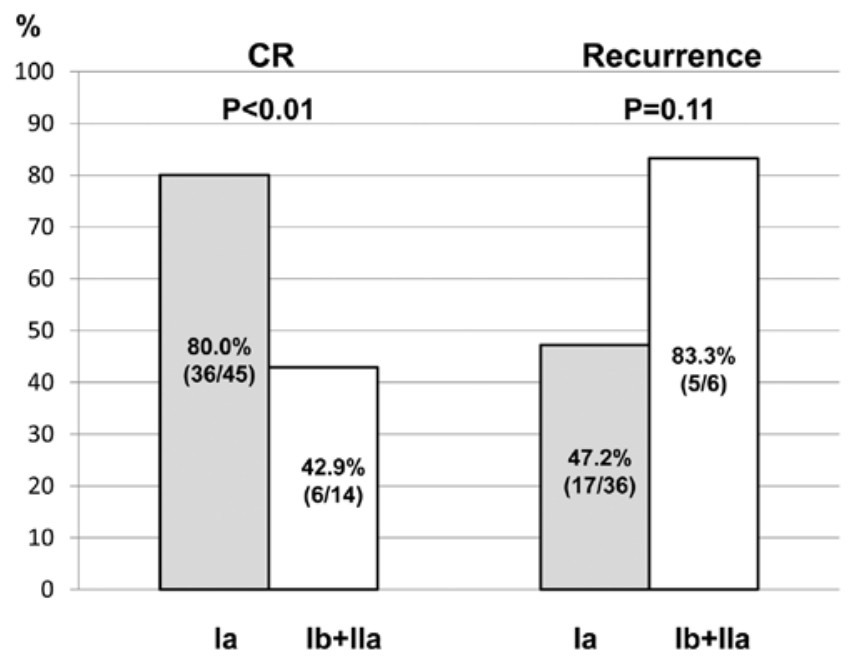

Figure 3. Initial CR rate and rate of recurrence following $\mathrm{CR}$ in relation to clinical stage. CR, complete response.

14 patients (54\%), however, pathological stages were more advanced in the remaining 10 patients (38\%). In 2 patients $(8 \%)$, the grade as ascertained by biopsy prior to MPA therapy differed from the postoperative grade; grade G1 prior to MPA therapy, but a final postoperative diagnosis of G2 and G3 in each patient, respectively.

As mentioned earlier, double cancer was confirmed in 2 cases (ovarian and tubal cancer), with an overall frequency of $3.3 \%$. In the 2 cases, residual endometrioid adenocarcinoma was not observed in the excised uterus.

Other than mild body weight increases, no adverse reactions were observed with the present treatment. No venous thrombosis was recorded in the patients.

\section{Discussion}

The therapeutic results and prognosis of fertility-sparing treatment using MPA for EC were reviewed retrospectively. Table II summarizes findings from past studies. While differences 
Table II. Summary of studies on patients treated with progestins.

\begin{tabular}{|c|c|c|c|}
\hline Author/Year (Refs.) & Number of cases & Complete response (\%) & Recurrence (\%) \\
\hline Kim et al, 1997 (9) & 7 & $4(57)$ & $2(50)$ \\
\hline Randall et al, 1997 (10) & 12 & $9(75)$ & 0 \\
\hline Imai et al, 2001 (11) & 15 & $8(53)$ & $3(37.5)$ \\
\hline Kaku et al, 2001 (12) & 12 & $9(75)$ & $2(22)$ \\
\hline Wang et al, 2002 (13) & 9 & $8(89)$ & $4(50)$ \\
\hline Niwa et al, 2004 (14) & 12 & $12(100)$ & $8(67)$ \\
\hline Ushijima et al, 2007 (15) & 39 & $26(67)$ & $14(54)$ \\
\hline Yamazawa et al, 2007 (16) & 9 & $7(78)$ & $2(29)$ \\
\hline Hahns et al, 2009 (17) & 35 & $22(63)$ & $9(41)$ \\
\hline Present study & 59 & $42(71)$ & $22(52)$ \\
\hline Total & 209 & $147(70)$ & $66(45)$ \\
\hline
\end{tabular}

exist among institutions in terms of response rates (range, $53-92 \%$ ) and recurrence rates (range, 11-53\%), the present findings were comparable. Our response rate exceeded $70 \%$, and 19 of the patients (32\% of enrolled patients) gave birth to a total of 25 children. This suggests that the present treatment is sufficient for the preservation of fertility.

However, patients and doctors require sufficient understanding prior to the initiation of treatment that the recurrence rate for this fertility-preserving treatment is high. As surgery is the standard therapy for EC, the present treatment should be performed only after obtaining written informed consent. As for the preoperative criteria for patient selection, the present study found differences in the rates of initial remission and the rate of recurrence after remission between stages Ia and more advanced stages, and therapeutic results for stages Ia were markedly improved. These results suggest that fertility-sparing treatment for EC should be limited to patients with stages up to Ia.

Our data suggest that for the first 2 years after treatment, patients should be followed up relatively frequently, approximately every 1-3 months, to check for recurrence. As cancer recurred after a long period of remission in certain cases, patients should be followed up for longer than after the first 2 years. Cases have been documented in which EC recurred after more than 10 years (18) or after childbirth (19). In the present study, EC recurred 5 years and 5 months after treatment in one patient ( 3 years and 10 months after childbirth). Thus, the possibility of recurrence is likely even after several years of remission, as well as after childbirth. These findings suggest that total hysterectomy can be performed as a preventive measure in females in remission who have given birth and do not want to have more children.

An investigation of patients who underwent surgery for recurrence revealed that preoperative clinical stages differed from actual pathological stages in certain cases. Disease stages may have advanced during therapy or preoperative investigations might have missed myometrial invasion. Several studies have revealed the limitations of preoperative staging of EC $(8,20)$. These limitations should be clearly explained to patients prior to therapy, and if a patient does not respond to therapy, the possibility that the stage is more advanced than Ia should be considered. In certain cases, the grade advanced from G1 to G2 or G3 postoperatively. Ota et al (21) documented grade progression from G1 to G2 in patients, who were unresponsive to MPA and underwent total hysterectomy. Thigpen et al (22) reported that higher grade is associated with lower MPA response rate. As MPA is a long-term treatment, periodic pathological tests are required during follow-up. If grades advance, termination of pharmacotherapy requires consideration versus continuing MPA. As abovementioned, the only patient who succumbed to disease following the present treatment began therapy at stage Ib of G1 endometrioid adenocarcinoma. Surgery was performed due to lack of response to MPA, but by the time of surgery the tumor had advanced to G3 endometrioid adenocarcinoma and had metastasized to the pelvic lymph nodes (pT1cN1M0). Kothari et al (23) also reported a patient in whom cancer recurred after fertility-sparing treatment, categorized as stage IV at the time of surgery. Patients need to consider that the present treatment is strictly optional, and that cancer stage can advance during or after MPA.

EC patients who are $\leq 40$ years old are susceptible to complications of ovarian and peritoneal cancer (24). In the present study, patients developing ovarian or tubal cancer were documented. Throughout regular follow-up, sufficient examination of the adnexa of the uterus is required. Furthermore, when totally excising the uterus by radical surgery, the aforementioned types of cancer should be considered when deciding whether to resect the bilateral adnexa, and informed consent must be obtained.

In general, symptoms, including metrorrhagia and postmenopausal bleeding, are observed in a number of EC patients. However, in the present study, approximately $60 \%$ of young EC patients who received fertility-sparing treatment had metrorrhagia. Additionally, in approximately $30 \%$ of patients, EC was accidentally identified during visits to a hospital for infertility, even though the patient had been asymptomatic during fertility tests. We have already reported that fertility tests detected EC in young females at a higher rate than those for EC in Japanese patients (25). In the group of patients who received fertility-sparing treatment in the present study, EC was detected by fertility tests in a number of patients, suggesting that fertility tests provide an opportunity for the 
early detection of EC. Healthcare professionals who conduct fertility tests should remain aware that they are dealing with patients at high risk of EC.

By retrospectively investigating fertility-sparing treatment using MPA for EC, the results and characteristics of patient backgrounds were established. The response rate was high, and the present treatment was considered acceptable for the purpose of enabling patients to give birth. However, the rate of recurrence is also high, thus results remain less effective when compared to surgery, the standard therapy for EC. In addition, standard treatment methods, including daily dose and administration period, have yet to be established. At present, treatments are administered various doese at different institutions. Thus, more studies are required for a standardized treatment to be established.

\section{References}

1. Jemal A, Siegel R, Xu J and Ward E: Cancer statistics. CA Cancer J Clin 60: 277-300, 2010.

2. Amant F, Moerman P, Neven P, Timmerman D, Van Limbergen E and Vergote I: Endometrial cancer. Lancet 366: 491-505, 2005.

3. Parazzini F, La Vecchia C, Bocciolone L and Franceschi S: The epidemiology of endometrial cancer. Gynecol Oncol 41: 1-16, 1991.

4. Brinton LA, Berman ML, Mortel R, et al: Reproductive, menstrual, and medical risk factors for endometrial cancer: results from a case-control study. Am J Obstet Gynecol 167: $1317-1325,1992$

5. Lurain JR: Uterine Cancer. In: Berek \& Novak's Gynecology, Berek JS (ed). 14th edition. Lippincott Williams \& Wilkins, Philadelphia, pp.1343-1401, 2007.

6. Crissman JD, Azoury RS, Barnes AE and Schellhas HF: Endometrial carcinoma in women 40 years of age or younger. Obstet Gynecol 57: 699-704, 1981.

7. Matsuda T, Marugame T, Kamo K, Katanoda K, Ajiki W, Sobue T, Japan Cancer Surveillance Research Group: Cancer incidence and incidence rates in Japan in 2005: based on data from 12 population-based cancer registries in the Monitoring of Cancer Incidence in Japan (MCIJ) project. Jpn J Clin Oncol 41: 139-147, 2011.

8. Gadducci A, Spirito N, Baroni E, Tana R and Genazzani AR: The fertility-sparing treatment in patients with endometrial atypical hyperplasia and early endometrial cancer: a debated therapeutic option. Gynecol Endocrinol 25: 683-691, 2009.

9. Kim YB, Holschneider CH, Ghosh K, Nieberg RK and Montz FJ: Progestin alone as primary treatment of endometrial carcinoma in premenopausal women. Report of seven cases and review of the literature. Cancer 79: 320-327, 1997.

10. Randall TC and Kurman RJ: Progestin treatment of atypical hyperplasia and well-differentiated carcinoma of the endometrium in women under age 40. Obstet Gynecol 90: 434-440, 1997.
11. Imai M, Jobo $T$, Sato $R$, Kawaguchi $M$ and Kuramoto $H$ : Medroxyprogesterone acetate therapy for patients with adenocarcinoma of the endometrium who wish to preserve the uterus-usefulness and limitations. Eur J Gynaecol Oncol 22: 217-220, 2001.

12. Kaku T, Yoshikawa H, Tsuda H, et al: Conservative therapy for adenocarcinoma and atypical endometrial hyperplasia of the endometrium in young women: central pathologic review and treatment outcome. Cancer Lett 167: 39-48, 2001.

13. Wang CB, Wang CJ, Huang HJ, et al: Fertility-preserving treatment in young patients with endometrial adenocarcinoma. Cancer 94: 2192-2198, 2002.

14. Niwa K, Tagami K, Lian Z, Onogi K, Mori H and Tamaya T: Outcome of fertility-preserving treatment in young women with endometrial carcinomas. BJOG 112: 317-320, 2005.

15. Ushijima K, Yahata H, Yoshikawa H, et al: Multicenter phase II study of fertility-sparing treatment with medroxyprogesterone acetate for endometrial carcinoma and atypical hyperplasia in young women. J Clin Oncol 25: 2798-2803, 2007.

16. Yamazawa K, Hirai M, Fujito A, et al: Fertility-preserving treatment with progestin, and pathological criteria to predict responses, in young women with endometrial cancer. Hum Reprod 22: 1953-1958, 2007.

17. Hahn HS, Yoon SG, Hong JS, et al: Conservative treatment with progestin and pregnancy outcomes in endometrial cancer. Int J Gynecol Cancer 19: 1068-1073, 2009.

18. Gotlieb WH, Beiner ME, Shalmon B, et al: Outcome of fertility-sparing treatment with progestins in young patients with endometrial cancer. Obstet Gynecol 102: 718-725, 2003.

19. Mitsushita J, Toki T, Kato K, Fujii S and Konishi I: Endometrial carcinoma remaining after term pregnancy following conservative treatment with medroxyprogesterone acetate. Gynecol Oncol 79: 129-132, 2000

20. Kinkel K: Pitfalls in staging uterine neoplasm with imaging: a review. Abdom Imaging 31: 164-173, 2006.

21. Ota T, Yoshida M, Kimura M and Kinoshita K: Clinicopathologic study of uterine endometrial carcinoma in young women aged 40 years and younger. Int J Gynecol Cancer 15: 657-662,2005.

22. Thigpen JT, Brady MF, Alvarez RD, et al: Oral medroxyprogesterone acetate in the treatment of advanced or recurrent endometrial carcinoma: a dose-response study by the Gynecologic Oncology Group. J Clin Oncol 17: 1736-1744, 1999.

23. Kothari R, Seamon L, Cohn D, Fowler J and O'Malley DM: Stage IV endometrial cancer after failed conservative management: a case report. Gynecol Oncol 111: 579-582, 2008.

24. Zivanovic O, Carter J, Kauff ND and Barakat RR: A review of the challenges faced in the conservative treatment of young women with endometrial carcinoma and risk of ovarian cancer. Gynecol Oncol 115: 504-509, 2009.

25. Fujiwara H, Ogawa S, Motoyama M, et al: Frequency and characteristics of endometrial carcinoma and atypical hyperplasia detected on routine infertility investigations in young women: a report of six cases. Hum Reprod 24: 1045-1050, 2009. 